Veresiuk T., Selskyy P. Comparative analysis of histological and morphometric changes of the arterial bed of the hind limbs of the rats in acute ischemia-reperfusion and correction with carbacetam. Journal of Education, Health and Sport. 2020;10(7):445-455. eISSN 2391-8306. DOI http://dx.doi.org/10.12775/JEHS.2020.10.07.046

https://apcz.umk.pl/czasopisma/index.php/JEHS/article/view/JEHS.2020.10.07.046

https://zenodo.org/record/4362487

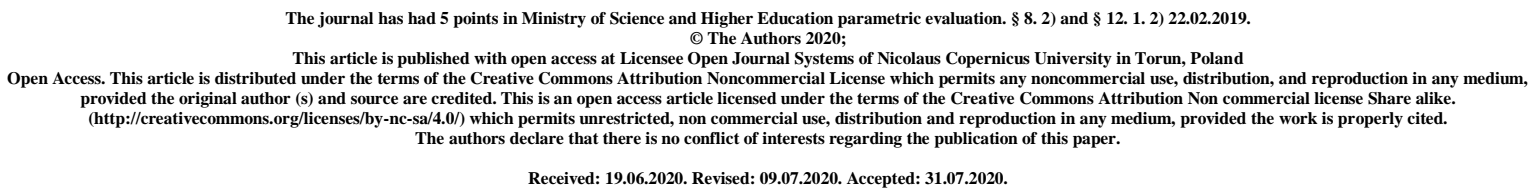

UDC 616.137-018:616-005.4-036.11:615.214.3]-092.9

\title{
COMPARATIVE ANALYSIS OF HISTOLOGICAL AND MOPHROMETRIC CHANGES OF THE ARTERIAL BED OF THE HIND LIMBS OF THE RATS IN ACUTE ISCHEMIA-REPERFUSION AND CORRECTION WITH CARBACETAM
}

\author{
T. Veresiuk, P. Selskyy
}

\section{Horbachevsky Ternopil National Medical University Ministry of Health of Ukraine}

\section{Abstract}

Resume. Ischemic-repefusion injury is a complex multifactorial tissue damage as the result of restoration of blood circulation, after the period of ischemia or lack of the oxygen, accompanied by the local and systemic disorders. Recently, more and more attention in treatment and prevention of postischemic disorders is paid to nootropic medicine, which smooths the phenomena of hypo- and hyperperfusion, and also improves tissue microcirculation.

The aim of the study was to establish the manifestations of morpho-functional remodeling of the vascular bed of the hind limbs of the rats during ischemia-reperfusion and under conditions of carbacetam correction using histological and morphometric investigations methods.

Materials and methods. There was histological and morphometric examination of the arterial bed of the hind limbs of 30 rats under conditions of ischemia-reperfusion injury (group I) and 30 rats in the simulation of ischemia-reperfusion injury, which in the post- 
ischemic period was administered carbacetam once a day $(5 \mathrm{mg} / \mathrm{kg}$ ) for 14 days (group II) was performed. There were 6 intact animals in the control group.

Simulation of ischemia was performed by applying SWAT rubber tourniquets on the hind limbs for 2 hours, and reperfusion - by removing the tourniquet. The animals were divided into 5 subgroups with reperfusion terms after 1, 2 hours and 1 day, as well as after 7 and 14 days. Histological examination was performed according to generally accepted methods. The vascular bed in the middle third of the thigh and shin, below the level of the application of the tourniquet was examined by Bresser Trino Researcher 40x $-1000 \mathrm{x}$ microscope. The morphometric parameters were calculated using SEO Image Lab software from Sumy Electron Optics.

Research results and their discussion. Analyzing of the obtained results, it was founded that after 1 hour of the repefusion the structural changes became systemic, and after 1 day they were the most significant. Histologically, the elastic membranes were thinned and torned up, the swelling became a total character. In the late reperfusion period, a gradual return of morphological changes to the initial state and the increased in the proliferative activity of the fibroblasts were revealed. Under the conditions of correction after 1 day, the positive dynamics became more pronounced and reached its maximum after 7 days of the study. Morphometrically, the differences between the groups are especially pronounced 7 days after the removal of the tourniquet. At the level of the femoral artery revealed a lower value of external $\left(D_{1}\right)$ by $1.9 \%(p<0,005)$ and internal $\left(D_{2}\right)$ diameters by $3,0 \%(p<0,005)$ and an increased in Vi by 3,54\% $(\mathrm{p}<0,005))$ in the specified experimental period in the animals with the correction, compared with the group without it.

In small-caliber arteries, on the contrary, there was a less pronounced vasoconstriction level, when applying the correction, because the value of $\mathrm{T}$ decreased by $5,4 \%(\mathrm{p}<0,005)$ and Vi by $20,28 \%(p<0,005)$, but there were higher indicator of $D_{1}$ by $1,89 \%(p<0,005)$ and $D_{2}$ by $11.55 \%(\mathrm{p}<0,005)$ compared to the group of animals without the correction.

Conclusions. Ischemia and repfusion cause vascular remodeling after 1 hour with a peak of manifestations after 1 day of reperfusion. Under the conditions of the correction, the acceleration of the remodeling with stabilization of the process and the most possible structural restoration after 7 days of the study was noted. Morphometric data indicate less pronounced vasodilation at the level of large caliber arteries and spasm of arteries of muscular branches during that experimental period in the group with correction.

Key words: artery; remodeling; ischemia-reperfusion; elastic membranes; carbacetam 
Introduction. Ischemic-reperfusion lesion, arising at restoration of arterial blood supply, underlies in conditions of acute or chronic ischemia $[5,7,11]$. Arterial ischemia accounts for about $10 \%$ of all gunshot wounds in the structure of combat trauma [1]. In $67 \%$ of cases of vascular damage at the prehospital stage, a tourniquet is applied, which can induce many complications, including ischemic-reperfusion injury, which occurs during the restoration of blood circulation and is characterized by severe flow and high mortality, which reaches $70 \%$, among the survivors, $20 \%$ face to the risk of high limb amputation $[1,2,3,5,9$, $10,11]$.

The literature data indicates that ischemia and reperfusion is a complex multifactorial process of damage and dysfunction of primary ischemic tissues as the result of restoration of arterial blood flow [5]. At present, there are local and systemic levels of reperfusion complications [5, 7]. It should be noted that if the study of systemic disorders is given enough attention, the local changes, which primarily depend on the incidence of reperfusion lesions are studied less intensively $[7,8]$.

To date, there are several mechanisms that can eliminate or mitigate the effects of reperfusion injury [7]. Recently, more and more attention is paid to nootropic medicines, their feature are that - they have a pronounced antihypoxic property and improve microcirculation in ischemic tissues [6]. We focused on the endogenous modulator of the GABAbenzodiazepine receptor complex, a derivative of B-carboline $[4,12]$ - carbacetam. The effectiveness of its usage has been repeatedly demonstrated in work on the restoration of cognitive impairment in traumatic brain injury and reduction of endogenous intoxication and oxidative stress in polytrauma [12].

In this study, the results of carbacetam usage were analyzed in order to study the local correction of the remodeling of the arterial bed of the hind limbs of rats during ischemia and reperfusion.

The aim of the study was to establish the manifestations of morpho-functional remodeling of the vascular bed of the hind limbs of the rats in ischemia-reperfusion and under conditions of correction with carbacetam using histological and morphometric methods.

Materials and methods Histological and morphometric examinations of the vascular bed of 60 rats were performed. There were 6 intact animals in the control group.

Simulation of ischemia was performed by applying rubber tourniquets SWAT (Stretch-Wrap-And-Tuck) on the right hind limb of the animal at the level of the inguinal fold for 2 hours under thiopental-sodium anesthesia. The reperfusion syndrome was modeled by 
removing the tourniquet and restoring blood circulation in the previously ischemic limb 2 hours after application. Observations of animals were carried out for 14 days.

During the experiment, the experimental animals were divided into three groups:

- the first experimental group - the animals with simulation of ischemic-reperfusion damage (30 rats);

- the second experimental group - the animals with simulation of ischemic-reperfusion damage, in which in reperfusion period 1-oxo-3,3,6-trimethyl-1,2,3,4-tetrahydroindolo quinolone (carbacetam) was administered intraperitoneally, at a dose of $5 \mathrm{mg}$ per kilogram of body weight, 1 time a day for 14 days of reperfusion period (30 rats);

- control group - intact animals (6 rats);

The animals of the first and the second experimental groups were divided into 5 subgroups, 6 animals each. The model of the early post-ischemic period in both groups was presented by subgroups of the animals with reperfusion changes after 1, 2 hours and 1 day, and the model of the late reperfusion period - by subgroups of animals 7 and 14 days after removal of the tourniquet.

All studies were conducted in compliance with the main provisions of the Law of Ukraine № 3447-IV of 21.02.06 "On the protection of animals from cruel treatment" (2006) and Council of Europe Directive 2010/63 EU on animal experiments.

After euthanasia and decapitation under thiopental-sodium anesthesia (500 $\mathrm{mg} \mathrm{x} \mathrm{kg}^{1}$ ), biological material was collected, which after dehydration and paraffin removal, dewaxing were stained with hematoxylin and eosin, picrofuchsin by Van Gieson's method, resorcin fuchsin by Weigert's elastic stain method, and Heidenhain's AZAN trichrome stain method [1].

The study of the materials was performed using a Bresser Trino Researcher 40x 1000 x microscope (serial number 0913137). The most demonstrative histological specimens were photographed with Digital Camera for Microscope Science Lab DCM 820 Resolution 8.0 Mp. The studied morphometric parameters were calculated using SEO Image Lab software from Sumy Electron Optics.

Results. Histological examination of the limbs vascular bed after 1 hour of repefusion showed that histological changes became systemic and manifested at all levels of structural organization of the vascular wall. After 2 hours, more pronounced manifestations of structural changes were noted. Endothelial cells were became rounded due to edema and exfoliated in layers. The intima thickened, lost its integrity and peeled off. The edema of the subendothelial layer increased. Elastic membranes split, straightened and fragmented, which we regarded as 
a sign of loss of vascular wall tone. Smooth myocytes were separated by intercellular substance. Vacuoles appeared in the cells. The edema dislocated the outer shell, infiltrated by lymphocytes, thus visually thickening the walls of blood vessels.

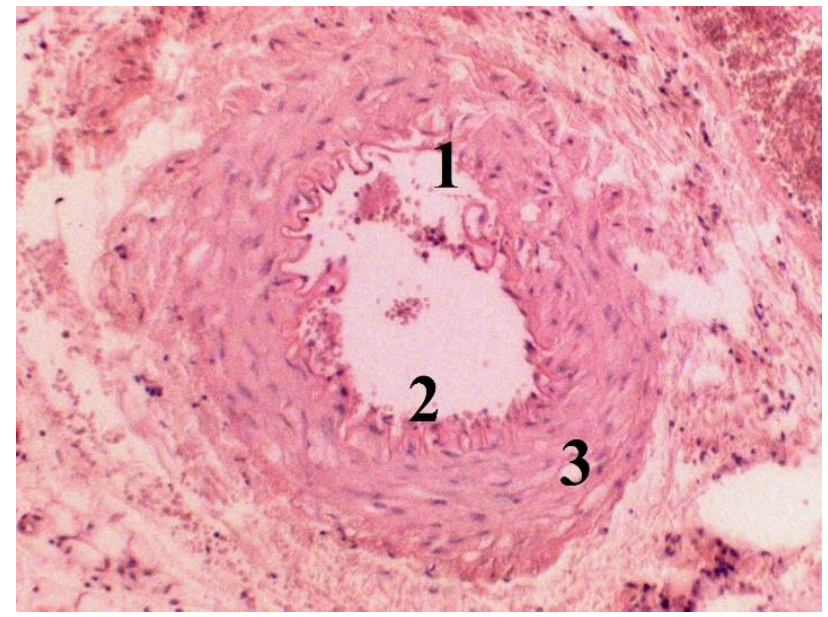

Fig.1. Desquamation of the endotheliocytes together with partially exfoliation of intima. Vacuoles dystrophy of the media's smooth myocytes. A section of the femoral artery 2 hours after reperfusion. Hematoxylin and eosin staining, magnification $\times 100$

Note. 1) detachment of intima, 2) desquamation of endothelial cells, 3) vacuolar dystrophy of smooth myocytes.

Structural changes of vessels became the most significant after 1 day of the experiment. Elastic membranes thinned and developed, the clarity of their layered structure was lost. Dystrophic changes of smooth myocytes were manifested by vacuolation with focal or total cytolysis. Circular cell infiltration is present in all layers, with parietal aggregation of erythrocytes and thrombosis in the vascular lumen. Swelling acquired a total character and spread to perivascular spaces and skeletal muscles (Fig. 2).

Histological changes of vessels after 7 days were generally similar to changes in the early reperfusion period with a decrease in swelling and restoration of endothelial coverage of the intima. The condition of the elastic frame also improved, but in response to ischemic influences, adventitia and perivascularly present a pronounced proliferation of collagen fibers.

After 14 days, the number of collagen fibers in the subendothelial space and outer shell - increased. The leiomyocyte hypertrophy was detected in the thickened media. The outer membrane fibers were fuzzy or absent. Swelling, desquamation and dystrophic changes of individual smooth myocytes were preserved. In all wall membranes, the intensity of lymphohistiocytic infiltration was significantly reduced, but the proliferative activity of fibroblasts was preserved (Fig. 3). 


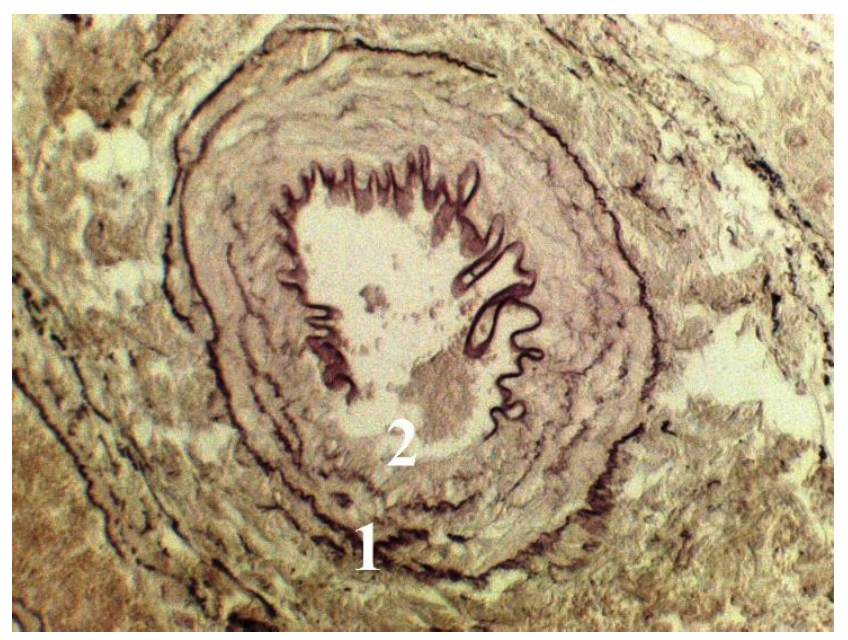

Fig. 2. Destructive changes in the arterial's walls are combined with intense inflammatory reaction, severe edema around the vessel with spread to the muscle. Section of the femoral artery after 1 day of perfusion, increase $\times 100$

Note. 1) deformation of the outer membrane, 2) lysis of the inner elastic membrane.

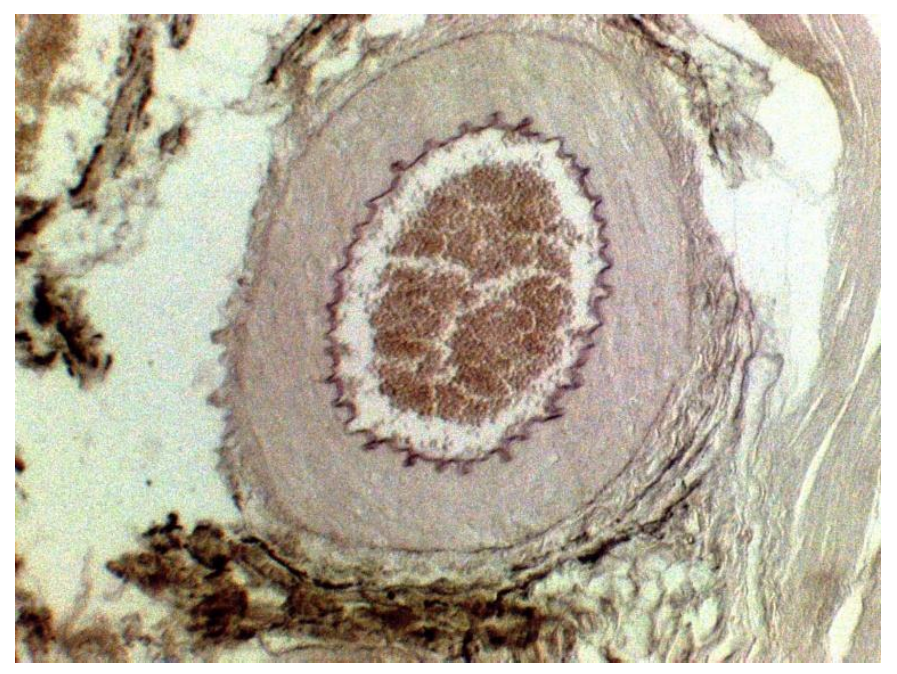

Fig. 3. The inner elastic membrane of the tibia artery of the rat after 14 days of reperfusion. The outer elastic membrane is weakly and unevenly contoured. Weigert's elastic stain, magnification $\times 100$

The morphological studies of the vascular bed under the conditions of carbacetam correction showed that after 1 hour the systemic disorders did not differ much from the ischemia-reperfusion period we had already detected at the same time, without the use of correction.

After 2 hours, the positive dynamics of the structural changes in the arteries was became more pronounced. The intensity and prevalence of edema, endothelial dysfunction 
and destruction of the elastic skeleton were decreased. The condition of smooth myocytes improved - the number of cells with vacuolated sarcoplasm were decreased. The intercellular amorphous substance was poorly differentiated. There are single lymphocytes, macrophages and fibroblasts in all wall membranes (Fig. 4).

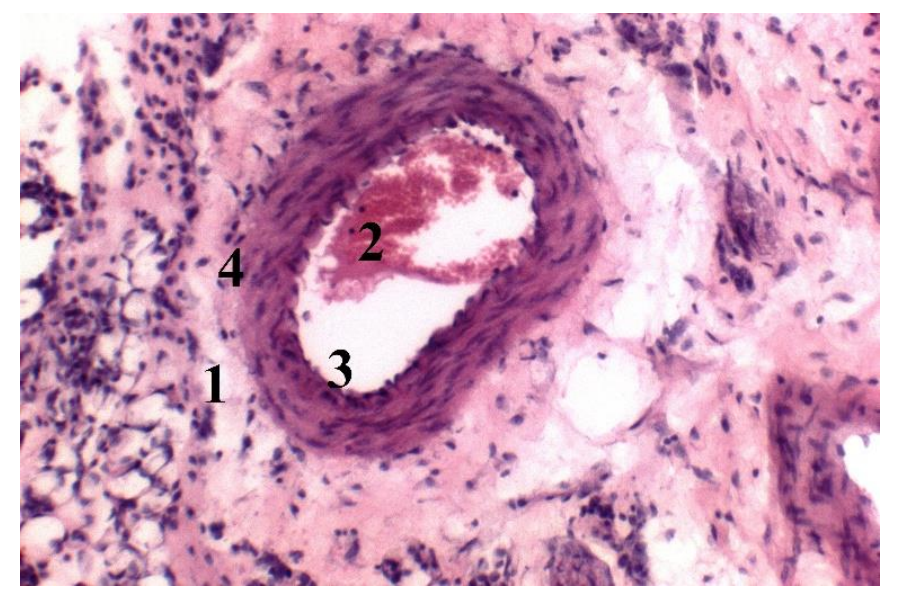

Fig. 4. The Foci of endothelial desquamation with erythrocyte adhesion. Perivascular edema and round cell stroma infiltration. The popliteal artery of the rat after 2 hours of reperfusion with correction. Staining with hematoxylin and eosin, magnification $\times 100$

Note 1) perivascular edema, 2) erythrocyte adhesion, 3) endothelial desquamation, 4) round cell infiltration.

After 1 day, the positive dynamics came and reached its maximum after 7 days of the study, slowing down and stabilizing after 14 days of the experiment. During these periods, we found a gradual restoration of endothelial coverage of the intima. The walls of the arteries acquired their inherent thickness. Elastic membranes restored the characteristic configuration. It should be noted that at the final stage of the study, we detected residual effects. Thus, part of the smooth myocytes of the arteries were hypertrophied along with the atrophic and increasing number of thin collagen fibers in the extracellular matrix, primarily on the border with the outer shell. In adventitia, the share of collagen fibers increased (fig. 5).

Ischemia and reperfusion under conditions of correction cause remodeling of the vascular bed, but its intensity was slightly lower compared to changes in morphometric parameters of the group without the use of correction. As can be seen from table 1, under conditions of correction, the values of the external and internal vascular diameters of the femoral artery $\left(D_{1}\right.$ and $\left.D_{2}\right)$ decreased in all experimental groups. The biggest difference was found after 7 days of the experiment, where the indicator $D_{1}$ by $1,9 \%(p<0,005)$ and $D_{2}$ by 
$3,0 \%(\mathrm{p}<0,005)$ decreased compared with similar indicators of groups, which were not corrected.

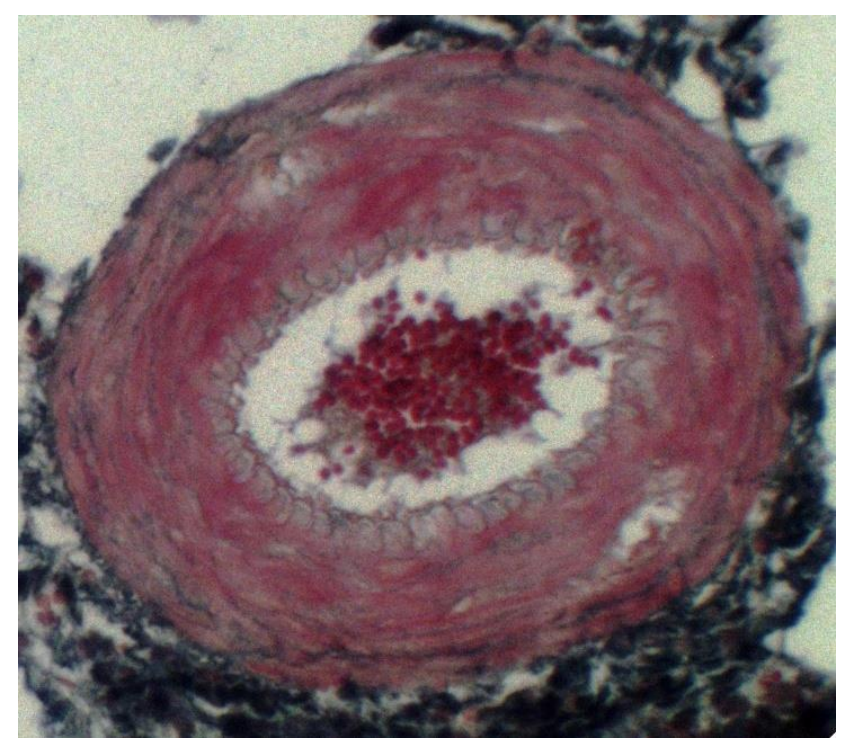

Fig. 5. Proliferation of collagen fibers in the outer shell and perivascular stroma. In the media, moderate collagenization of its outer layer. Rat's tibia artery after 14 days of reperfusion with correction. Azan staining according to Heidenhain, magnification $\times 100$

The thickness of the vascular wall of the femoral artery (T) in both groups did not change and ranged from $1 \%(\mathrm{p}>0,05)$. However, the value of the Vogenvort index (VI) index of femoral artery increased by $1,77 \%(\mathrm{p}<0,005)$ - after 1 day, by $3,54 \%$ - after 7 days ( $p<0,005)$ and by $2,21 \%$ - after 14 days $(p<0,005)$ compared with similar indicators of the group of animals without the use of correction. Differences between other groups were not so significant and did not exceed $1 \%(\mathrm{p}>0,05)$.

As can be seen from table 1, in the animals with correction showed a predominance of the outer and inner diameters of the vascular wall of muscle branches $\left(D_{1}\right.$ and $\left.D_{2}\right)$ in all experimental groups, but the greatest difference was found after 7 days of the experiment, where $\mathrm{D}_{1}$ exceeded by $1,7 \%(\mathrm{p}<0,005)$, and $\mathrm{D}_{2}$ by $11,55 \%(\mathrm{p}<0,005)$ similar values of groups without correction.

The wall thickness (T) and the Vogenvort index (VI) of the muscular branches vessels in the conditions of correction decreased, and most clearly these changes were manifested 7 days after the restoration of blood circulation, being by $5,4 \%(p<0,005)$ and $20,28 \%$ $(p<0,005)$ lower compared to the group without the introduction of carbacetam. 
Table 1 - Morphometric parameters of the outer diameter $\left(D_{1}\right)$, the inner diameter $\left(\mathrm{D}_{2}\right)$, the wall thickness $(\mathrm{T})$, Vogenvort index (VI) of the artery of the hind limb of the rats in reperfusion period after removal of the arterial tourniquet and during their correction with carbacetam $(\mathrm{M} \pm \mathrm{m})$

\begin{tabular}{|c|c|c|c|c|c|}
\hline \multirow[t]{2}{*}{ Artery } & \multirow{2}{*}{$\begin{array}{c}\text { Reperfusion } \\
\text { after }\end{array}$} & \multicolumn{4}{|c|}{ Parameters } \\
\hline & & D & $\mathrm{D}_{2}, \mathrm{mkm}$ & $\mathrm{T}, \mathrm{m}$ & VI \\
\hline Femoral & Control & $612,54 \pm 10,44$ & $360,82 \pm 6,32$ & $125,86 \pm 3,71$ & $189,02 \pm 7,42$ \\
\hline \multirow[t]{2}{*}{ Femoral } & 1 hour & $646,21 \pm 9,64^{*}$ & $393,12 \pm 8,57^{*}$ & $126,54 \pm 1,13$ & $171,05 \pm 4,36$ \\
\hline & + correction & $644,26 \pm 9,61^{*}$ & $391,94 \pm 8,54^{*}$ & $126,16 \pm 1,13$ & $171,04 \pm 4,36$ \\
\hline \multirow{2}{*}{ Femoral } & 2 hours & $654,09 \pm 7,02^{* *}$ & $400,74 \pm 5,20^{* *}$ & $126,68 \pm 2,48$ & $166,90 \pm 5,00^{*}$ \\
\hline & + correction & $650,49 \pm 6,98^{* *}$ & $397,66 \pm 5,16^{* *}$ & $126,42 \pm 2,47$ & $168,08 \pm 5,00^{*}$ \\
\hline \multirow{2}{*}{ Femoral } & 1 day & $657,04 \pm 12,54^{*}$ & $404,84 \pm 7,14^{* *}$ & $126,10 \pm 4,56$ & $164,22 \pm 7,55^{*}$ \\
\hline & + correction & $651,12 \pm 12,43^{*}$ & $399,01 \pm 7,04^{* *}$ & $126,06 \pm 4,53$ & $167,12 \pm 7,63^{*}$ \\
\hline \multirow{2}{*}{ Femoral } & 7 days & $628,15 \pm 15,63$ & $376,86 \pm 8,60$ & $126,15 \pm 6,92$ & $181,74 \pm 12,96$ \\
\hline & + correction & $616,21 \pm 15,33$ & $364,58 \pm 8,34^{* * *}$ & $125,82 \pm 6,78$ & $188,17 \pm 13,26^{*}$ \\
\hline \multirow{2}{*}{ Femoral } & 14 days & $621,16 \pm 13,74$ & $369,03 \pm 7,34$ & $126,07 \pm 5,31$ & $184,62 \pm 10,22$ \\
\hline & + correction & $613,09 \pm 13,57$ & $361,64 \pm 7,19$ & $125,72 \pm 5,24$ & $188,70 \pm 10,37$ \\
\hline $\begin{array}{l}\mathrm{Mu} \\
\text { bran }\end{array}$ & Control & $43,85 \pm 0,80$ & 22,0 & $11,91 \pm 0,33$ & $336,09 \pm 16,11$ \\
\hline \multirow{2}{*}{$\begin{array}{l}\text { Muscle } \\
\text { branches }\end{array}$} & 1 hour & $43,90 \pm 1,30$ & $18,90 \pm 0,51^{* *}$ & $12,50 \pm 0,45$ & $440,79 \pm 15,96^{*}$ \\
\hline & + correction & $43,98 \pm 1,31$ & $19,19 \pm 0,52^{* *}$ & $12,40 \pm 0,45$ & $426,52 \pm 15,54^{*}$ \\
\hline \multirow{2}{*}{$\begin{array}{l}\text { Muscle } \\
\text { branches }\end{array}$} & 2 hours & $42,58 \pm 1,24$ & $16,17 \pm 0,51^{* * / *}$ & $13,20 \pm 0,40^{*}$ & $596,19 \pm 18,55^{*}$ \\
\hline & + correction & $42,96 \pm 1,25$ & $16,70 \pm 0,53^{* * / *}$ & $13,13 \pm 0,40^{*}$ & $564,22 \pm 17,70^{*}$ \\
\hline \multirow{2}{*}{$\begin{array}{l}\text { Muscle } \\
\text { branches }\end{array}$} & 1 day & $42,36 \pm 0,85$ & $15,76 \pm 0,38^{* *}$ & $13,30 \pm 0,30^{* *}$ & $626,60 \pm 23,48^{*}$ \\
\hline & + correction & $42,74 \pm 0,86^{*}$ & $16,37 \pm 0,40^{* *}$ & $13,18 \pm 0,30^{*}$ & $585,25 \pm 22,14^{*}$ \\
\hline \multirow{2}{*}{$\begin{array}{l}\text { Muscle } \\
\text { branches }\end{array}$} & 7 days & $44,51 \pm 1,02$ & $19,14 \pm 0,74^{* * * *}$ & $12,68 \pm 0,18^{*}$ & $446,91 \pm 17,57^{*}$ \\
\hline & + correction & $45,35 \pm 1,04$ & $21,36 \pm 0,83^{* * * *}$ & $12,00 \pm 0,16$ & $356,29 \pm 14,66^{*}$ \\
\hline \multirow{2}{*}{$\begin{array}{l}\text { Muscle } \\
\text { branches }\end{array}$} & 14 days & $4 J, 39 \pm 1,02$ & $21,23 \pm 0,55^{\text {**** }}$ & $12,09 \pm 0,31$ & $359,46 \pm 12,85^{*}$ \\
\hline & + correction & $45,63 \pm 1,03$ & $21,76 \pm 0,56$ & $11,93 \pm 0,31$ & $341,65 \pm 12,35$ \\
\hline
\end{tabular}

Notes: $*$ - $\mathrm{p}<0,05$ compared to the control group; $* *$ - $\mathrm{p}<0,01$ compared to the control group; $* * *-\mathrm{p}<0,05$ compared to the previous group. 


\section{Conclusions}

1. Ischemia and reperfusion cause remodeling of the arterial bed of the hind limbs of the rats with the peak of the largest histological changes 1 day after the restoration of blood supply.

2. The structural abnormalities in the early reperfusion period included edematous syndrome and destructive changes in vessels with an inflammatory response to injury. The initiating factor was ischemic endothelial damage and subsequent manifestations of endothelial dysfunction. In the late reperfusion period, the remodeling was characterized mainly by the increased in the activity of the fibroblastic differon and synthesis of the connective tissue matrix in the vessel walls and perivascular.

3. The morphometric data confirm that the differences between the groups are especially pronounced 7 days after removal of the tourniquet. At the level of the femoral artery revealed a lower value of the external $\left(D_{1}\right)\left(\right.$ by $1,9 \%(p<0,005)$ and the internal $\left(D_{2}\right)$ (by $3,0 \%(\mathrm{p}<0,005)$ diameters and an increase in $\mathrm{Vi}$ by $3,54 \%(\mathrm{p}<0,005)$ in the specified experimental period in the animals with correction.

4. In small-caliber arteries, on the contrary, there was a less pronounced vasoconstriction when applying the correction, because the value of $\mathrm{T}$ decreased by $5,4 \%$ ( $p<0,005$ ) and Vi by 20,28\% ( $p<0,005$ ), but there was a higher value of the $\mathrm{D}_{1}$ (by $1,89 \%$ $(\mathrm{p}<0,005)$ ) and the $\mathrm{D}_{2}$ (by $11,55 \%(\mathrm{p}<0,005)$ ) compared to the uncorrected group of the animals.

5. Histologically, under the condition of correction, there is a consistent acceleration of remodeling with stabilization of the process and the most possible structural restoration after 7 days of the experiment.

6. Morphometrically, the differences between the groups are especially pronounced 7 days after removal of the tourniquet with an increase in VI by $3,54 \%(p<0,005)$ at the level of the femoral artery, indicating less pronounced vasodilation of large arteries and its reduction by $20,28 \%(p<0,005)$ at the level of muscle branches, which indicates the decrease in vasoconstriction of small caliber arteries, whereas in the animals without correction morphological and morphometric signs of recovery of the arterial bed were detected after 14 days.

\section{References}

1. Dick, F., Li, J., Giraud, M.-N., Kalka, Ch., Schmidli, J., Tevaearai, H. (2008). Basic control of reperfusion effective against reperfusion injury in a realistic rodent model of acute 
limb ischemia. Cardiovascular Surgery, 118(19), 1920-1928. doi:10.1161/CIRCULATIONAHA.108.787754

2. Dunn, J. C., Kusnezov, N., Schoenfeld, A. J., Orr, J. D., Cook, P. J., \& Belmont Jr, P. J. (2016). Vascular injuries in combat-specific soldiers during operation Iraqi freedom and operation enduring freedom. Annals of Vascular Surgery, 35, 30-37. doi: 10.1016/ j.avsg.2016.01.040

3. Elsharawy, M. A., Elsaid, A., \& Elsharawi, I. (2014). Reperfusion of Delayed Acute Occlusive Limb Ischemia: Is It Worthwhile? World Journal of Cardiovascular Diseases, 4(12), 580-585. doi: 10.4236/wjcd.2014.412070

4. Gamzin, S. S., Alekseeva, L. V., \& Lapina, G. P. (2015). Methodological aspects of biochemical and pharmacological analysis of the effect of nootropics on the peptidergic system and metabolism in general. Bulletin of Tver State University. Series: Biology and Ecology, (3), 40-52.

5. Henyk, S. M., \& Symchych, A. V. (2016). Reperfusion syndrome after revascularization of lower extremity ischemia. Heart and Blood Vessels, (3), 104-108.

6. Hubka, V. O., Konovalenko, I. A., \& Suzdalenko, O. V. (2015). The results of treatment of patients with acute arterial ischemia of the extremities. Pathology, 2(34), 55-58.

7. Malchenko, O. A. (2016). Pathogenetic substantiation of approaches to the correction of limb tissue damage in experimental reperfusion syndrome. (Dis. Ph. D.). Kyiv.

8. Osmanova, A. A. (2010). Dynamics of the microcirculatory bed under compression trauma of the limbs soft tissue and correction by infusion Perftoran. (Dis. Ph. D.). Makhachkala.

9. Rudolf, R. (2017). Avoiding long-term muscle damage upon ischaemia-reperfusion. Acta Physiologica, 219(2), 343-345. doi: 10.1111/apha.12769

10. Tran, Th. P., Tu, H., Pipinos, I. I., Muelleman, R. L., Albadawi, H., Li, Y-L. (2011). Tourniquet-induced acute ischemia-reperfusion injury in mouse skeletal muscles : involvement of superoxide. European Journal of Pharmacology, 650 (1), 328-334. doi: 10.1016/j.ejphar.2010.10.037

11. Zasimovich, V. N., \& Ioskevich, N. N. (2017). Reperfusion reoxygenation syndrome as a problem of reconstructive surgery of arteries in chronic ischemia of the lower extremities of atherosclerotic genesis. Surgery News, 25(6), 632-642.

12. Ziablitsev, S. V., Starodubska, O. O., Bohza, S. L. (2017). Pharmacological correction of cognitive disorders in traumatic brain injury. Actual Problems of Modern Medicine. Bulletin of VSEIU "Ukrainian Medical Dental Academy", 17(3(59)), 25-29. 